\title{
Electromagnetic Low-Intensity Extracorporeal Shock Wave Therapy in Patients with Erectile Dysfunction: A Sham-Controlled, Double-Blind, Randomized Prospective Study
}

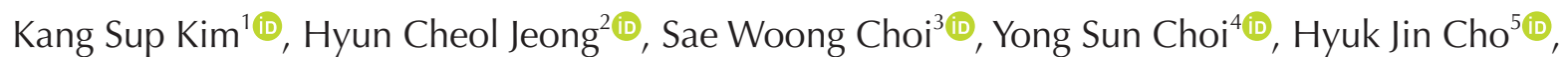 \\ U-Syn Ha ${ }^{5}$ (i) Sung-Hoo Hong ${ }^{5}$ (i) , Ji Youl Lee ${ }^{5}$, Seung Wook Lee ${ }^{6}$, Sun Tae Ahn ${ }^{7}$, Du Geon Moon ${ }^{7}$, \\ Woong Jin Bae ${ }^{5,8}$ (i), Sae Woong Kim ${ }^{5,8(\mathbb{D})}$ \\ ${ }^{1}$ Department of Urology, Incheon St. Mary's Hospital, College of Medicine, The Catholic University of Korea, ${ }^{2}$ Department of Urology, \\ Kangdong Sacred Heart Hospital, Hallym University College of Medicine, ${ }^{3}$ Department of Urology, Yeouido St. Mary's Hospital, College \\ of Medicine, The Catholic University of Korea, ${ }^{4}$ Department of Urology, Eunpyeong St. Mary's Hospital, College of Medicine, The Catholic \\ University of Korea, ${ }^{5}$ Department of Urology, Seoul St. Mary's Hospital, College of Medicine, The Catholic University of Korea, ${ }^{6}$ Department \\ of Urology, Hanyang University College of Medicine, ${ }^{7}$ Department of Urology, Korea University College of Medicine, ${ }^{8}$ Catholic Integrative \\ Medicine Research Institute, College of Medicine, The Catholic University of Korea, Seoul, Korea
}

Purpose: The aim of the present study was to evaluate the efficacy and safety of the electromagnetic-type low-intensity extracorporeal shock wave therapy (Li-ESWT) in patients with erectile dysfunction (ED).

Materials and Methods: The randomized, sham-controlled, double-blind prospective study was performed at two referral hospitals. Participants were randomized in a 1:1 ratio to receive sham or Li-ESWT for 6 weeks. ED was evaluated at screening and at 4 and 7 weeks after treatment. Participants were asked to complete the international index of erectile function-erectile function (IIEF-EF) domain questionnaire, erection hardness scale (EHS), and sexual encounter profile questionnaire (SEPQ 2 and 3). The development of complications was investigated.

Results: Eighty-one of 96 patients completed the study. The median change in the IIEF-EF score in the Li-ESWT and sham groups was 5.1 and -2.2 ( $\mathrm{p}<0.001)$, respectively, at the 7 -week follow-up; 47.4\% (18/38) patients had EHS $<3$, of which $77.8 \%(14 / 18)$ showed significant improvement in virtue of Li-ESWT treatment $(\mathrm{p}=0.001)$. A significant improvement was observed in the percentage of 'Yes" responses to SEPQ 2 and 3 in the Li-ESWT group vs. sham group from baseline to 7-week follow-up (91.3\% vs. $69.4 \%$; $\mathrm{p}=0.008$ and $50.0 \%$ vs. $14.3 \%$; $\mathrm{p}=0.002$, respectively). No patients reported pain or other adverse events during treatment or follow-up.

Conclusions: Thus, Li-ESWT could have a role in improving erectile function. Furthermore, it is safe. We believe that Li-ESWT is an attractive new treatment modality for patients with ED.

Keywords: Electromagnetic; Erectile dysfunction; Humans; Low-intensity extracorporeal shock wave therapy

This is an Open Access article distributed under the terms of the Creative Commons Attribution Non-Commercial License (http://creativecommons.org/licenses/by-nc/4.0) which permits unrestricted non-commercial use, distribution, and reproduction in any medium, provided the original work is properly cited.

Received: Sep 26, 2019 Revised: Oct 31, 2019 Accepted: Oct 31, 2019 Published online Nov 19, 2019

Correspondence to: Sae Woong Kim (iD https://orcid.org/0000-0002-9127-9400

Department of Urology, Seoul St. Mary's Hospital, College of Medicine, The Catholic University of Korea, 222 Banpo-daero, Seocho-gu, Seoul 06591, Korea.

Tel: +82-2-2258-6226, Fax: +82-2-599-7839, E-mail: ksw1227@catholic.ac.kr

Correspondence to: Woong Jin Bae (iD https://orcid.org/0000-0001-7703-1161

Department of Urology, Seoul St. Mary's Hospital, College of Medicine, The Catholic University of Korea, 222 Banpo-daero, Seocho-gu, Seoul 06591, Korea.

Tel: +82-82-2-2258-6229, Fax: +82-82-2-599-7839, E-mail: bwoong@catholic.ac.kr 


\section{INTRODUCTION}

Erectile dysfunction (ED) is a common disease among middle-aged men. ED is defined as the continuous inability to retain and maintain penile erection sufficient to permit sexual performance [1,2]. First-line treatment modalities for ED are systemic medications, such as phosphodiesterase type 5 (PDE 5) inhibitors, which have been available and used for more than two decades. If this treatment fails, vacuum pump, intraurethral medication, penile injection, and penile implants are available [3]. However, these treatments do not follow a curative approach, and the results are not always satisfactory. Furthermore, they do not modify the underlying pathophysiology of the erectile mechanism.

After its use as a treatment modality for urinary tract stones [4], shock wave therapy, considered minimally invasive with proven safety and convenience, has been used for ischemic heart disease and musculoskeletal disease [5,6]. Recently, low-intensity extracorporeal shock wave therapy (Li-ESWT) to the corpora cavernosa was adopted as a treatment option for ED [7]. Although the complete mechanism of Li-ESWT in ED has not been elucidated, it is attributed to stimulation of angiogenesis and improvement in penile blood flow [8,9]. Furthermore, Li-ESWT induces stimulation of the expression of angiogenesis-related growth factor, recruitment of endothelial progenitor cell, and activation of Schwann cell, which has the ability for nerve regeneration $[10,11]$.

In this study, we evaluated the efficacy and safety of Li-ESWT as a treatment modality for ED. To our knowledge, this is the first Korean prospective, randomized, placebo-controlled clinical study to assess the efficacy and safety after Li-ESWT in men with ED. We hypothesized that Li-ESWT would have a beneficial effect in treating ED.

\section{MATERIALS AND METHODS}

\section{Ethics statement}

This sham-controlled, double-blind, randomized prospective study was conducted in accordance with the ethical guidelines of the Declaration of Helsinki. This study protocol was reviewed and approved by the Institutional Review Board of The Catholic University of Korea (approval number: KC17DNDE0474) after approval by the Korean Ministry of Food and Drug Safe- ty. Informed consent was obtained from all patients when they were enrolled for the study.

\section{Study population}

During 2017 to 2018, 96 patients with ED were included in this sham-controlled, double-blind, randomized, prospective study and followed for 7 weeks. This study was performed at 2 referral hospitals in Korea. Patients who visited the hospitals for treatment of ED were enrolled as cohorts. All patients who met the criteria for participation provided a written informed consent before initiating Li-ESWT. Patients who were already on PDE 5 inhibitors were instructed to take a 4 -week washout period before randomization. Furthermore, patients who participated in this study were not allowed to consume PDE 5 inhibitors during the study period. Detailed medical history, physical examination, and ED-related questionnaires were administered to the subjects. After completing a baseline measurement visit, participants (1:1) were allocated to the sham group or Li-ESWT group using stratified block randomization method. We enrolled: clinically diagnosed mild or moderated ED patients for at least 6 months according to the National Institute of Health Consensus statement, patient age $\geq 20$ years, and a stable sexual relationship for $>3$ months. Patients who met any of the following conditions were excluded from the present study: severe $\mathrm{ED}$, psychogenic $\mathrm{ED}$, neurological pathology, prior radical prostatectomy, rectal extirpation, radiation therapy to the pelvic area, no anatomical malformation, heart disease inhibiting sexual contact, and medication with anticoagulant.

\section{Interventions}

The treatments were executed using the MT $2000 \mathrm{H}$ (Urontech Korea, Hwaseong, Korea), which utilizes an electromagnetic system to generate shock waves, which are focused using a parabolic reflector to an energy maximum inside the tissue. A water-based gel was used to enhance conductivity. All patients were scheduled to receive two Li-ESWT sessions every week for a period of 3 weeks that were repeated after a 3-week resting period. Four and 7 weeks after completion of the procedure, participants were assessed by international index of erectile function-erectile function (IIEF$\mathrm{EF}$ ) domain score, which was calculated by comparing the total scores of erection hardness scale (EHS), sexual encounter profile questionnaire 2 (SEPQ 2; Were you 
able to insert your penis into your partner's vagina?) and 3 (SEPQ 3; Did your erection last long enough for you to have successful intercourse?), and any adverse event. ED severity is classified into five categories according to scoring system of IIEF-EF score: no ED (score 26-30), mild (22-25), mild to moderate (17-21), moderate (11-16), and severe (6-10) [12].

Each Li-ESWT treatment session consisted of 3,000 shock waves applied to the penis with a frequency of 5 $\mathrm{Hz}$ in doses of 1,000 shock waves with energy densities of $20 \mathrm{~mJ} / \mathrm{mm}^{2}, 15 \mathrm{~mJ} / \mathrm{mm}^{2}$, and $12 \mathrm{~mJ} / \mathrm{mm}^{2}$, applied to the root of the penis, to the shaft, and at a few millimeters proximal to the glans, respectively. For the sham treatment, the same probe as that in Li-ESWT was used, except the energy was set to 0 during each treatment, and a similar noise was delivered to the patients during the procedure.

Before each treatment, the patients were asked if they had experienced any side effects since the previous consultation, and they were encouraged to verbalize any discomfort during the sessions. In addition, all patients were instructed to contact the research team if they experienced any side effects during the procedure.

\section{Outcome parameters}

The primary objective was to evaluate the 7 -week change from baseline in the IIEF-EF domain score, which was used to assess ED at the urologic clinic after termination of Li-ESWT. The secondary outcome measured was to assess the 7-week change from baseline in the EHS and SEPQ 2 and 3 scores. Furthermore, we evaluated the 4-week change from baseline for IIEF-EF domain score, SEPQ 2 and 3, and EHS.

\section{Statistical analysis}

Given the information from several evidences [13]. where Li-ESWT could be effective to patients with $\mathrm{ED}$, we calculated the target sample size $(n=74)$ using $G^{*}$ power software (ver. 3.1) with one tailed test, 1:1 allocation, $\beta=0.8$, and $\alpha$ error $=0.05$.

The descriptive statistics for continuous variables were presented as mean and standard deviation, and the normal distribution of the data was tested. Independent t-test or Wilcoxon's rank sum test was used to compare the two groups. For categorical variables, the number and percentage of subjects were presented and compared between the two groups using the chi-square test or Fisher's exact test. The $\mathrm{p} \leq 0.05$ was considered statistically significant. All statistical analyses were executed using SAS ver. 9.3 (SAS Institute, Cary, NC, USA).

\section{RESULTS}

A total of 96 participants were considered for enrollment. One patient declined to participate in this study. Ninety-five subjects who met the inclusion and exclusion criteria were allocated to two groups. A total of 38 patients were randomized into the Li-ESWT arm, and 43 patients were randomized into the sham therapy arm. Six patients in Sham group and five patients in Li-ESWT group were dropped for withdrawal of consent during treatment period. Two patients in sham group and one patient withdrew their consent during 7 weeks follow-up. Therefore, fourteen participants were dropped for withdrawal of consent during this study (Fig. 1). Patient's demographics and baseline characteristic are presented in Table 1. There were no significant differences in baseline IIEF-EF score and baseline EHS between the two groups.

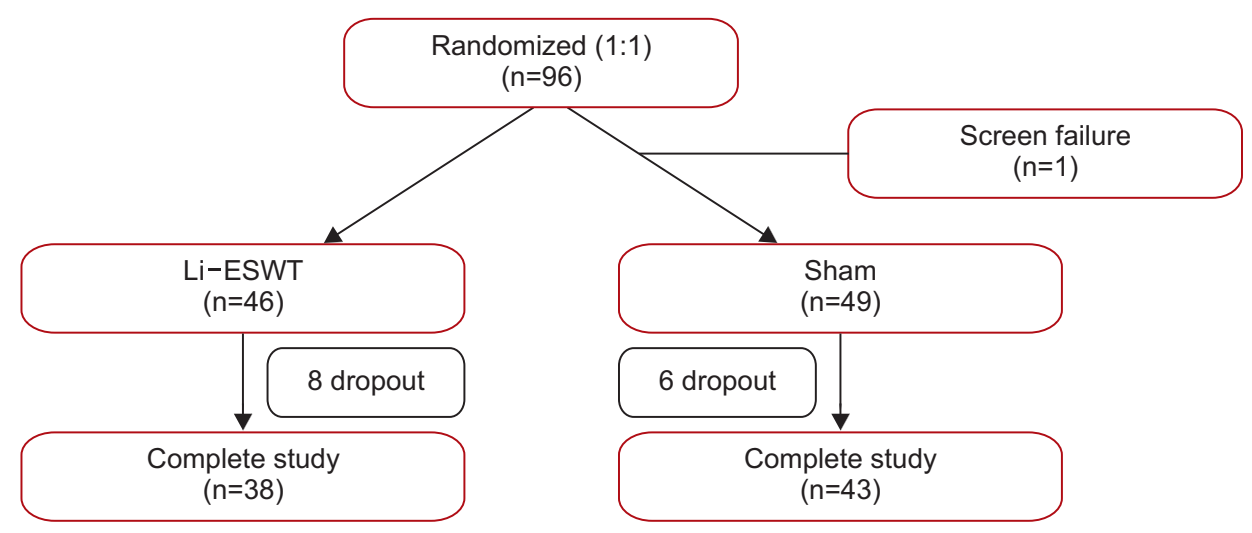

Fig. 1. Subject disposition. Li-ESWT: low-intensity extracorporeal shock wave therapy. 
The baseline IIEF-EF domain score in the Li-ESWT and sham groups were $16.6 \pm 3.0$ and $16.7 \pm 3.2$, respectively ( $p>0.99$ ) IIEF-EF domain score increased significantly from baseline to 7 weeks ( $16.6 \pm 3.0$ at baseline and $21.7 \pm 8.2$ at 7 weeks, $\mathrm{p}<0.001)$ within the Li-ESWT group compared to that in the sham group (16.7 \pm 3.2 at baseline and $14.5 \pm 5.4$ at 7 weeks) (Fig. $2 \mathrm{~A}$ ). The median change in IIEF-EF domain score in the Li-ESWT and sham groups was 5.1 and -2.2 , respectively $(\mathrm{p}<0.001)$ at the 7-week follow-up (Fig. 2B).

Table 1. Participants' demographics and characteristics

\begin{tabular}{lccl}
\hline \multicolumn{1}{c}{ Variable } & $\begin{array}{c}\text { Li-ESWT } \\
\text { group }(\mathbf{n}=38)\end{array}$ & $\begin{array}{c}\text { Sham group } \\
(\mathbf{n}=43)\end{array}$ & p-value \\
\hline Mean age (y) & $63.2 \pm 5.4$ & $65.1 \pm 7.9$ & 0.637 \\
BMI $\left(\mathrm{kg} / \mathrm{m}^{2}\right)$ & 24.9 & 24.8 & 0.488 \\
Incidence of ED risk factor & & & 0.35 \\
$\quad$ Hypertension & 18 & 17 & \\
$\quad$ Diabetes & 9 & 9 & \\
$\quad$ Vascular disease & 7 & 5 & \\
Mean baseline IIEF-EF & $16.6 \pm 3.0$ & $16.7 \pm 3.2$ & 1.0 \\
$\quad$ domain score & & & \\
Mean baseline EHS & $2.5 \pm 0.9$ & $2.5 \pm 0.8$ & 0.617 \\
Baseline SEPQ 2 (Yes) & $28(73.7)$ & $36(83.7)$ & 0.356 \\
Baseline SEPQ 3 (Yes) & $6(15.8)$ & $7(16.3)$ & 0.682 \\
\hline
\end{tabular}

Values are presented as mean \pm standard deviation, mean only, number only, or number (\%).

LI-ESWT: low-intensity extracorporeal shock wave therapy, BMI: body mass index, ED: erectile dysfunction, IIEF-EF domain: international index of erectile function-erectile function domain, EHS: erection hardness scale, SEPQ: sexual encounter profile questionnaire.
A total of $47.4 \%(18 / 38)$ of patients had EHS $<3$, and of these, $77.8 \%(14 / 18)$ showed improvement with LiESWT treatment at 7 weeks ( $\mathrm{p}=0.001$ ). Whereas, $34.9 \%$ (15/43) of patients had EHS <3 increased to $46.5 \%$ (20/43) after sham treatment at 7 weeks (Table 2). A statistically higher mean EHS for the Li-ESWT group compared to that for the sham group was noted in 7 weeks (3.1 \pm 0.6 vs. $2.4 \pm 0.9, \mathrm{p}=0.001$ ).

A significant improvement was seen in the percentage of "Yes" responses to SEPQ2 and 3 from the baseline in the Li-ESWT group compared to that in the sham group at the 7-week follow-up (91.3\% vs. 69.4\%; $\mathrm{p}=0.008$ and $50.0 \%$ vs. $14.3 \%$; $\mathrm{p}=0.002$, respectively).

Table 2. Comparison of patients between Li-ESWT and sham with EHS

\begin{tabular}{cccc}
\hline EHS & $\begin{array}{c}\text { Li-ESWT } \\
\text { group }(\mathbf{n}=38)\end{array}$ & $\begin{array}{c}\text { Sham } \\
\text { group }(\mathbf{n}=43)\end{array}$ & p-value \\
\hline $\begin{array}{c}\text { Baseline } \\
<3\end{array}$ & $18(47.4)$ & $15(34.9)$ & 0.194 \\
$\geq 3$ & $20(52.6)$ & $28(65.1)$ & \\
Week 4 & & & 0.003 \\
$<3$ & $5(13.2)$ & $20(46.5)$ & \\
$\geq 3$ & $33(86.8)$ & $23(53.5)$ & \\
Week 7 & & & 0.001 \\
$<3$ & $4(10.5)$ & $20(46.5)$ & \\
$\geq 3$ & $34(89.5)$ & $23(53.5)$ & \\
\hline
\end{tabular}

Values are presented as number (\%).

Li-ESWT: low-intensity extracorporeal shock wave therapy, EHS: erection hardness score.
IIEF-EF score at baseline, week 4 \& week 7

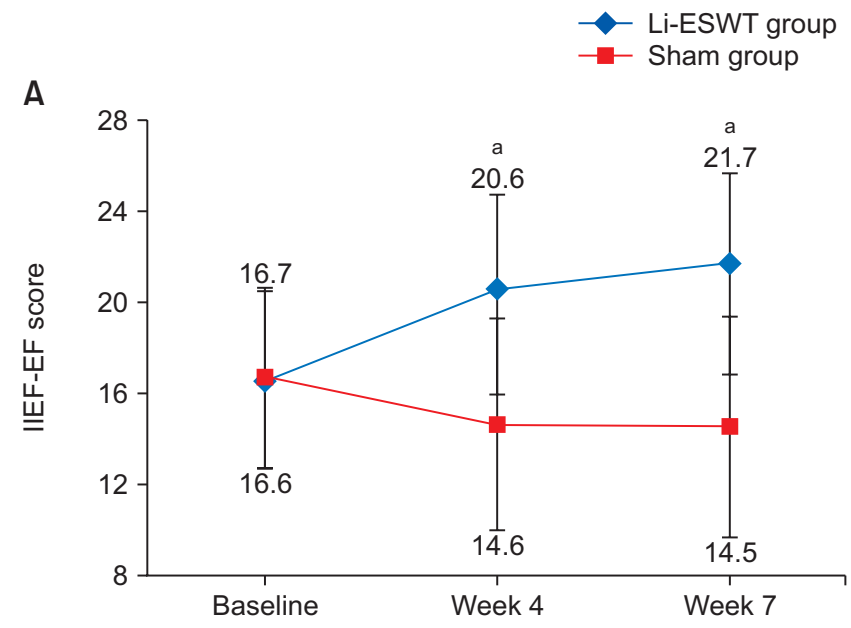

IIEF-EF mean scores changes from baseline

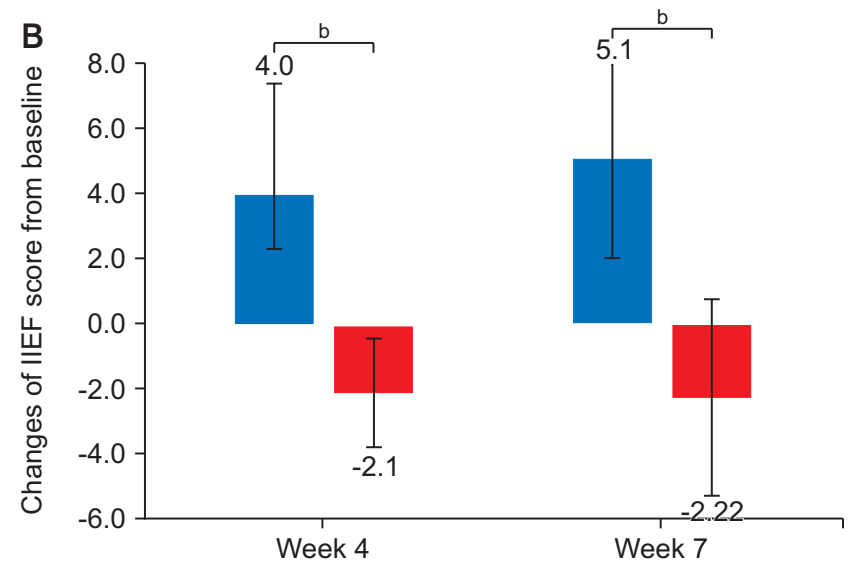

Fig. 2. (A) Mean international index of erectile function-erectile function (IIEF-EF) domain score for patients treated with low-intensity extracorporeal shock wave therapy (Li-ESWT) or sham treatment at baseline, week 4, and week 7. (B) Comparison of the mean IIEF-EF domain score changes from baseline. ${ }^{a} p<0.001$ compared with baseline. ${ }^{b} p<0.001$. 
Complications and side effects, such as pain, hematoma, hematuria, and bruising were assessed for the entire study duration. No adverse events were seen during Li-ESWT treatment and after the procedure.

\section{DISCUSSION}

Eighty-one of 96 patients completed the study. Significant change in the IIEF-EF score was seen in the Li-ESWT group than that in the sham group at the 7-week follow-up (5.1 vs. -2.2; p<0.001); more subjects showed significant improvement in EHS (47.4\% [18/38] vs. 77.8\% [14/18], $\mathrm{p}<0.001$ ); significant change in the percentage of "Yes" responses to SEPQ 2 and 3 was more in the Li-ESWT group $v s$. sham group from baseline to 7-week follow-up (91.3\% vs. $69.4 \%$; $\mathrm{p}=0.008$ and $50.0 \%$ vs. $14.3 \%$; $\mathrm{p}=0.002$, respectively). No patients reported pain or other adverse events during treatment or follow-up.

Although treatment modalities for ED, such as PDE 5 inhibitor, intracavernosal injection of vasoactive drugs, in vacuum constriction devices, and surgical implantation of penile prosthesis enhance sexual function and quality of erection, they are not curative modalities and do not resolve the underlying pathological changes in the penis. Recent studies suggest that LiESWT enhance spontaneous and natural penile erectile function by improving the underlying penile pathological changes through angiogenic properties and penile hemodynamics $[8,9,14]$.

Vardi et al [15] reported the effectiveness and safety of Li-ESWT in the treatment of ED. This study treated 20 patients twice per week for 3 weeks and repeated the procedure after a 3 -week period of rest. The authors suggested an improvement in the IIEF score at 1 month and persevered at 6 months. Furthermore, ten men did not use PDE 5 inhibitors after 6 months of follow-up. The same study group underwent a randomized double-blind, sham-controlled study [13]. The authors reported that Li-ESWT had a positive short-term clinical and physiologic effect on the erectile function of patients who responded to PDE 5 inhibitors. All patients did not experience discomfort and did not report any adverse effects from Li-ESWT. Recently, several systemic reviews and meta-analyses showed that LiESWT, as a non-invasive treatment modality, showed more improvement in the IIEF or IIEF-EF score compared to sham treatment $[7,16,17]$. The authors concluded that Li-ESWT significantly improved erectile function in patients with vasculogenic ED.

Our study group conducted experimental studies prior to the present clinical study for the time being to verify the effectiveness and stability of the MT2000H ESWT electromagnetic generator. In the electromagnetic technique, electric current is passed through a coil to generate a strong magnetic field. The amplitude of the focused waves rises non-linearly when the acoustic wave disseminates toward the target point [18]. A shock wave device and the coaxial configuration of an imaging device have the strong point of more accurate focusing than other types of devices, such as electrohydraulic and piezoelectronic. Jeong et al [19] reported the effect of LI-ESWT in an animal model of diabetes mellitus (DM) induced by an intraperitoneal injection of streptozotocin. The intracavernous pressure (ICP) in the DM+Li-ESWT group showed a significant increase compared to that in the DM group $(p<0.05)$. The vascular endothelial growth factor, endothelial nitric oxide synthase, neuronal nitric oxide synthase, and cyclic guanosine monophase expression level were recovered to levels in the DM group $(\mathrm{p}<0.05)$. We confirmed the effectiveness of an electromagnetic ESWT device using a DM-related ED model. After verifying the efficacy of the electromagnetic type ESWT, we performed animal experiments using combination therapy with mesenchymal stem cell therapy and Li-ESWT in a rat model of diabetic ED [20]. The study confirmed that combination of mesenchymal stem cell therapy and Li-ESWT showed better results than those using a single-treatment modality. The main mechanisms of combination treatment are increased expression of vascular endothelial growth factor, inducing vasodilation and angiogenesis by activating the nitric oxide/cyclic guanosine monophosphate signaling pathway. Furthermore, our study group analyzed the synergy efficacy of Li-ESWT with Korean herbal formula (KH-204) in an animal model of DM-induced ED [21]. Although the ESWT group, KH-204 group, and ESWT+KH 204 group showed significant increase in ICP compared with the DM group, the ESWT+KH-204 group showed statistically improved ICP compared with the singletreatment groups. Based on these positive experimental results of electromagnetic ESWT, we conducted this double-blind, randomized, prospective clinical study. In our study, all measured ED-related parameters, such as IIEF-EF domain score, EHS, and SEPQ 2 and 3 showed significant improvements in the Li-ESWT group than 
those in the sham group at the end of treatment and the follow-up duration. Li-ESWT seems to be safe and highly tolerable in this study. No patients reported penile pain, bruising, or hematuria during the procedure or at subsequent follow-up visits. The strength of this study is that it is the first sham-control, double-blind, randomized prospective study in South Korea using the electromagnetic ESWT machine.

We acknowledge some limitations in this present study. One limitation of the present study was that objective measurements such as penile hemodynamics were not executed. However, well-designed randomized control studies showed hemodynamic improvement measured via flow-mediated dilatation technique and supported the results of the present study [13,22]. Second, the European Association of Urology and the American Urological Association do not recommend the use of Li-ESWT for ED, except for investigation [3,23]. Therefore, it is mandatory to perform larger randomized controlled studies with the same population and adequate follow-up to assess the longevity and effectiveness of Li-ESWT effects. Third, follow-up evaluation was not executed after a certain period of time following completion of the procedure. Hence, we were unable to conclude whether the effect of ESWT to ED was maintained after treatment. Lastly, the number of patients in the present study was small; hence, we could not investigate the results according to the etiology and degree of ED.

\section{CONCLUSIONS}

This is the first double-blind, sham-controlled, randomized study that showed that Li-ESWT was effective and safe to improve erectile function in Korean men with ED. Further studies with long-term follow-up with better-defined targets for Li-ESWT and optimal therapeutic protocols are needed to assess the efficacy of Li-ESWT. Further clinical studies using combination treatment such as stem cell and natural product with Li-ESWT are needed to provide a new treatment modality for ED in the future.

\section{ACKNOWLEDGEMENTS}

This research was supported by a grant of the Korea Health Technology R\&D Project through the Korea Health Industry Development Institute (KHIDI), fund- ed by the Ministry of Health \& Welfare, Korea (grant HI17C1944).

\section{Conflict of Interest}

The authors have nothing to disclose.

\section{Data Sharing Statement}

The data analyzed for this study have been deposited in HARVARD Dataverse and are available at https://doi. org/10.7910/DVN/TNJMMQ.

\section{Author Contribution}

Conceptualization: KSK, WJB, SWK. Data curation: KSK, WJB, STA, DGM. Formal analysis: KSK, WJB, SWK. Investigation: KSK, WJB, SWK. Methodology: KSK, WJB, SWK. Project administration: KSK, WJB, SWK. Resources: KSK, WJB, STA, DGM. Supervision: HCJ, SWC, YSC, HJC, USH, SHH, JYL, SWL. Writing - original draft: KSK, WJB, SWK. Writing - review \& editing: KSK, WJB, SWK.

\section{REFERENCES}

1. NIH Consensus Conference. Impotence. NIH Consensus Development Panel on Impotence. JAMA 1993;270:83-90.

2. Rajfer J, Aliotta PJ, Steidle CP, Fitch WP 3rd, Zhao Y, Yu A. Tadalafil dosed once a day in men with erectile dysfunction: a randomized, double-blind, placebo-controlled study in the US. Int J Impot Res 2007;19:95-103.

3. Hatzimouratidis K, Amar E, Eardley I, Giuliano F, Hatzichristou D, Montorsi F, et al; European Association of Urology. Guidelines on male sexual dysfunction: erectile dysfunction and premature ejaculation. Eur Urol 2010;57:804-14.

4. Schuüller J, Chaussy C. [A new therapy for urinary calculi. Noninvasive kidney stone crushing]. Krankenpfl Soins Infirm 1984;77:30-1. German.

5. Al-Abbad H, Simon JV. The effectiveness of extracorporeal shock wave therapy on chronic achilles tendinopathy: a systematic review. Foot Ankle Int 2013;34:33-41.

6. Ito K, Fukumoto Y, Shimokawa H. Extracorporeal shock wave therapy for ischemic cardiovascular disorders. Am J Cardiovasc Drugs 2011;11:295-302.

7. Lu Z, Lin G, Reed-Maldonado A, Wang C, Lee YC, Lue TF. Low-intensity extracorporeal shock wave treatment improves erectile function: a systematic review and meta-analysis. Eur Urol 2017;71:223-33. 
8. Gruenwald I, Appel B, Kitrey ND, Vardi Y. Shockwave treatment of erectile dysfunction. Ther Adv Urol 2013;5:95-9.

9. Lei H, Liu J, Li H, Wang L, Xu Y, Tian W, et al. Low-intensity shock wave therapy and its application to erectile dysfunction. World J Mens Health 2013;31:208-14.

10. Li H, Matheu MP, Sun F, Wang L, Sanford MT, Ning H, et al. Low-energy shock wave therapy ameliorates erectile dysfunction in a pelvic neurovascular injuries rat model. J Sex Med 2016;13:22-32.

11. Nishida T, Shimokawa H, Oi K, Tatewaki H, Uwatoku T, Abe $\mathrm{K}$, et al. Extracorporeal cardiac shock wave therapy markedly ameliorates ischemia-induced myocardial dysfunction in pigs in vivo. Circulation 2004;110:3055-61.

12. Cappelleri JC, Rosen RC, Smith MD, Mishra A, Osterloh IH. Diagnostic evaluation of the erectile function domain of the International Index of Erectile Function. Urology 1999;54: 346-51.

13. Vardi Y, Appel B, Kilchevsky A, Gruenwald I. Does low intensity extracorporeal shock wave therapy have a physiological effect on erectile function? Short-term results of a randomized, double-blind, sham controlled study. J Urol 2012;187: 1769-75.

14. Aicher A, Heeschen C, Sasaki K, Urbich C, Zeiher AM, Dimmeler S. Low-energy shock wave for enhancing recruitment of endothelial progenitor cells: a new modality to increase efficacy of cell therapy in chronic hind limb ischemia. Circulation 2006;114:2823-30.

15. Vardi Y, Appel B, Jacob G, Massarwi O, Gruenwald I. Can low-intensity extracorporeal shockwave therapy improve erectile function? A 6-month follow-up pilot study in patients with organic erectile dysfunction. Eur Urol 2010;58:243-8.
16. Zou ZJ, Tang LY, Liu ZH, Liang JY, Zhang RC, Wang YJ, et al. Short-term efficacy and safety of low-intensity extracorporeal shock wave therapy in erectile dysfunction: a systematic review and meta-analysis. Int Braz J Urol 2017;43:805-21.

17. Sokolakis I, Hatzichristodoulou G. Clinical studies on low intensity extracorporeal shockwave therapy for erectile dysfunction: a systematic review and meta-analysis of randomised controlled trials. Int J Impot Res 2019;31:177-94.

18. Ogden JA, Tóth-Kischkat A, Schultheiss R. Principles of shock wave therapy. Clin Orthop Relat Res 2001;(387):8-17.

19. Jeong HC, Jeon SH, Qun ZG, Kim KS, Choi SW, Bashraheel F, et al. Effects of next-generation low-energy extracorporeal shockwave therapy on erectile dysfunction in an animal model of diabetes. World J Mens Health 2017;35:186-95.

20. Zhu GQ, Jeon SH, Bae WJ, Choi SW, Jeong HC, Kim KS, et al. Efficient promotion of autophagy and angiogenesis using mesenchymal stem cell therapy enhanced by the low-energy shock waves in the treatment of erectile dysfunction. Stem Cells Int 2018;2018:1302672.

21. Jeong HC, Bae WJ, Zhu GQ, Jeon SH, Choi SW, Kim SJ, et al. Synergistic effects of extracorporeal shockwave therapy and modified Ojayeonjonghwan on erectile dysfunction in an animal model of diabetes. Investig Clin Urol 2019;60:285-94.

22. Kitrey ND, Gruenwald I, Appel B, Shechter A, Massarwa O, Vardi Y. Penile low intensity shock wave treatment is able to shift PDE5i nonresponders to responders: a double-blind, Sham Controlled Study. J Urol 2016;195:1550-5.

23. Burnett AL, Nehra A, Breau RH, Culkin DJ, Faraday MM, Hakim LS, et al. Erectile dysfunction: AUA Guideline. J Urol 2018;200:633-41. 